\title{
Biomimetic routes for photosplitting of water
}

\author{
K K ROHATGI-MUKHERJEE, A B CHATTERJEE, B B BHOWMIK, \\ S C BERA, MANORAMA BHATTACHARYA, J CHAUDHURY and \\ B MANNA \\ Department of Chemistry, Jadavpur University, Calcutta 700 032, India
}

\begin{abstract}
Natural photosynthesis involves splitting of water into $\mathrm{O}_{2}$ and $\mathrm{H}_{2}$ by thermodynamically uphill electron transfer using photon energy absorbed by green chlorophylls and other light-absorbing molecules in leaf chloroplast. It is a multiphotonmultielectron-photosensitized reaction. The artificial photocatalytic system must mimic both the function and the scheme of natural photosynthesis, to bring about this uphill electron transfer. The key concept is organization to prevent back electron transfer. A preliminary report of the attempts made in our laboratory to promote vectorial electron transfer by using various techniques such as PEC cells and liposomes, using chlorophylls isolated from spinach leaves as well as synthesis of their analogues porphyrins synthesized in the laboratory is presented.
\end{abstract}

Keywords. Photosplitting of water; artificial photosynthesis; photo-induced electron transfer; liposomes; porphyrins; biomimetic routes.

\section{Introduction}

The mechanism of photosynthesis in plants, to convert simple molecules like $\mathrm{CO}_{2}$ and $\mathrm{H}_{2} \mathrm{O}$ into more complex molecules such as carbohydrates, has been designed by nature to store solar energy in the form of chemical energy. The overall reaction is photosensitized oxidation of water evolving $\mathrm{O}_{2}$ and fixation of $\mathrm{CO}_{2}$ in a number of dark steps, initiated by the reduced species generated in the photoact. The absorbed energy is collectively stored in the products, the reduced species, which could also be $\mathrm{H}_{2}$ in the presence of catalyst hydrogenase and the oxidized species such as $\mathrm{O}_{2}$. The energy is released when they combine again under appropriate conditions. The reduced species can therefore be called photofuels. Thus the primary event in photosynthesis is one-electron transfer reaction triggered by light absorption by the green chlorophylls, and involves sequential absorption of two photons by two photosystems which act as photosensitizers. This one-electron transfer reaction driven by photoexcited states of chlorophylls need multielectron steps to obtain the final products and seems to be a very promising photochemical reaction for production of 'solar fuels' such as hydrogen, ammonia, methane, carbon monoxide etc. if prototype of plant photosynthesis can be developed.

Thus we find that in plant photosynthesis sunlight is used as the driving force to convert low energy starting materials such as $\mathrm{H}_{2} \mathrm{O}$ to high energy product materials such as mixtures of $\mathrm{H}_{2}+\mathrm{O}_{2}$ in a series of endergonic reactions. The positive free energy $(\Delta G)$ is stored in the products.

The photosplitting of water is an endergonic reaction,

$$
\mathrm{H}_{2} \mathrm{O} \longrightarrow \mathrm{H}_{2}+\frac{1}{2} \mathrm{O}_{2}
$$

with thermodynamic parameters: $\Delta H=286.20 \mathrm{~kJ} \mathrm{~mol}^{-1}, \Delta G=245 \cdot 41 \mathrm{~kJ} \mathrm{~mol}^{-1}$ and $\Delta S=149.50 \mathrm{~J} \mathrm{~mol}^{-1} \mathrm{deg}^{-1}$. The radiation wavelength required to trigger this reaction can be calculated by the relation $h c / \lambda=\Delta G$ and corresponds to the red 
region of the spectrum. This wavelength is not absorbed by water. Therefore photochemical breakdown of water to $\mathrm{H}_{2}$ and $\mathrm{O}_{2}$ is not likely to be very efficient using visible light unless, like photosynthesis, two series-coupled photochemical reactions are used, one a photochemical reduction reaction to yield a strong reductant which can reduce water and the other a photochemical oxidation reaction to yield a strong oxidant which can oxidize water to $\mathrm{O}_{2}$ :

$$
\begin{aligned}
2 \mathrm{H}_{2} \mathrm{O} \stackrel{4 h r}{\longrightarrow} \mathrm{O}_{2}+4 \mathrm{H}^{+}+4 e^{-}, \\
2 e^{-}+2 \mathrm{H}_{2} \mathrm{O} \longrightarrow \mathrm{H}_{2}+2 \mathrm{OH}^{-} .
\end{aligned}
$$

Nature has devised mechanisms to couple these two multiphoton-multielectron reactions by forcing the electron to flow uphill from a region of low redox potential, $\mathrm{O}_{2} / \mathrm{H}_{2} \mathrm{O}=+0.82 \mathrm{~V}$ to a region of high redox potential $\mathrm{H}_{2} \mathrm{O} / \mathrm{H}_{2}=-0.41 \mathrm{~V}$, both with reference to $\mathrm{NHE}$ at $\mathrm{pH}=7$, against the thermodynamic gradient with the help of photon energy. Such a coupled system has not been produced as yet although Calvin and others have been working on it. The $\mathrm{H}_{2}$ evolution system is more or less understood but the $\mathrm{O}_{2}$ evolution system of photosynthesis has still eluded the researchers. The artificial photocatalytic system must mimic both the function and the scheme of natural photosynthesis, to bring about this uphill electron transfer.

The photocatalytic water cleavage technology is associated with the development of three stages of reaction:

(i) Pigment-sensitized photon-induced charge separation to produce strong oxidant and strong reductant:

$$
A+D \underset{\text { Sensitizer }}{\stackrel{h v}{\text { acceptor donor pigments }}} A^{-}+D^{+}
$$

Followed by dark reactions:

(ii) $2 \mathrm{~A}^{-}+2 \mathrm{H}_{2} \mathrm{O} \stackrel{\text { catalyst }}{\longrightarrow} 2 \mathrm{~A}+\mathrm{H}_{2}+2 \mathrm{OH}^{-}$

(iii) $4 \mathrm{D}^{+}+2 \mathrm{H}_{2} \mathrm{O} \longrightarrow$ catalyst $\longrightarrow 4 \mathrm{D}+\mathrm{O}_{2}+4 \mathrm{H}^{+}$

Both the dark reactions will need suitable catalysts for evolution of $\mathrm{H}_{2}$ and $\mathrm{O}_{2}$.

The major difficulty that arises in simulating natural photosynthesis is to accomplish the first step in such a fashion that the back recombination of charges, $D^{+}+A^{-} \longrightarrow$ $D+A$, is suppressed. This simple and highly exothermic reaction usually proceeds much faster than the catalytic reaction to split water. For this reason, photosensitized water cleavage is difficult to accomplish in homogeneous systems, more so because $\mathrm{O}_{2}$ evolution reaction needs 4 electrons collectively and $\mathrm{H}_{2}$ evolution 2 electrons. In homogeneous solution these steps may be identified as

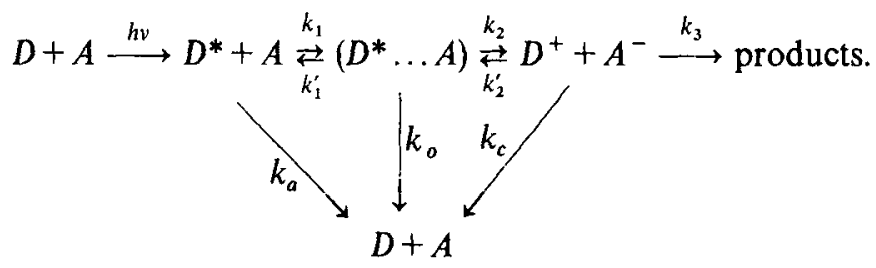

The rate constants $k_{a}, k_{o}$ and $k_{c}$ are for recombination reactions at various stages and must be minimised for efficient product formation. 
The natural photosynthesis involves two photosystems and very efficient heterogeneous membranes called thylakoid membrane. The organization of necessary components in the membrane brings about fast unidirectional pathways of electron and proton movements, thereby avoiding recombination reaction. Heterogeneity allows the organisation of reactants and products into compartments of given environments and facilitates transport of substrates to and from different locations. The key concept is organization (Calvin 1979; Fendler 1983, 1985).

The molecular assemblies resembling reaction centres of natural photosynthetic apparatus should consist of reactive centres in which the components $D$ and $A$ and consequently the components $D^{+}$and $A^{-}$formed during the reaction (1) are spatially separated for prevention of charge recombination and creating a photon gradient essential for photophosphorylation. Some of the various types of assemblies obtained artificially are presented in figure 1 . At the present state of art, microheterogeneous systems based on microemulsions, micellar solutions, membranes and lipid vesicles are considered to be more interesting systems for the development of structurally organized molecular photocatalytic devices. The oxidizing and reducing steps can be spatially separated in systems such as membranes and liposomes and can even be operated independently as in microemulsions or micelles. Semiconductor electrodes with suitable band gaps, set up in a photoelectrochemical cells have also been found useful since the electrodeelectrolyte interface, due to the development of space-charge region, can bring about efficient charge separation on photoexcitation. The two electrodes are coupled by electron flow through outer circuit and the flow of positive charge through the solution in opposite direction balancing the charge flow. By using the membrane between the two compartments, efficiency can be increased. Such systems can be used as transducers of light-to-electrical energy to generate photovoltage and
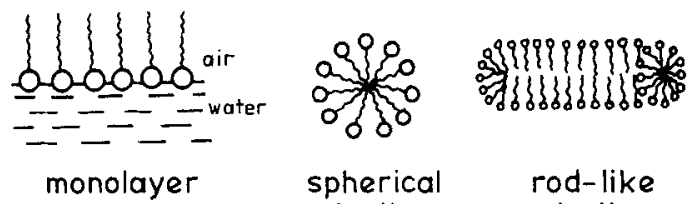

rod-like micelle

micelle
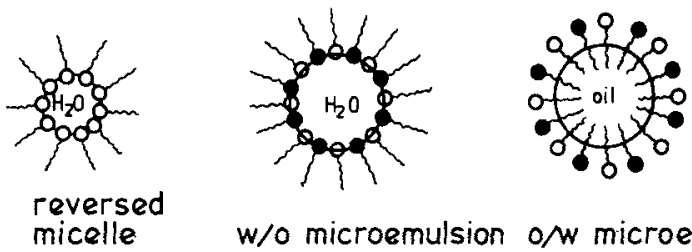

w/o microemulsion

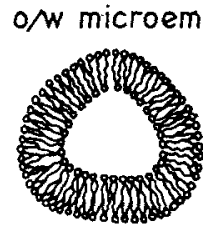

single

compartment

vesicle

Figure 1. Various forms of organized systems. 
photocurrents and also as light-to-chemical energy for storage as chemical compounds, depending on the redox couples used and potentials generated. The thermodynamic potential for decomposition of water to $\mathrm{H}_{2}$ and $\mathrm{O}_{2}$ is $1.23 \mathrm{~V}$ per molecule. The choice of semiconductor electrodes depends on their Fermi levels. Films of organic semiconductors deposited on suitable electrodes are also effective. For coupled systems, the two redox potentials, that of $\mathrm{O}_{2}$ evolving system and that of $\mathrm{H}_{2}$ evolving systems must encompass $-0.41 \mathrm{~V}$ and $+0.82 \mathrm{~V}$ range plus some overpotential. In homogeneous systems suitable relays have been used to wisk away electrons or fill up the positive hole generated by the initial excitation step.

A simple scheme for sensitized photolysis of water based on the use of two photochemical reactions coupled in series is presented in figure $2 \mathrm{a}$ using a membrane permeable to electron and $\mathrm{H}^{+}$ions. Figure $2 \mathrm{~b}$ represents the energetic scheme for solar energy utilization using two light absorbers such as semiconductor or dyes and requiring two photons of energies $h v_{1}$ and $h v_{2}$, to drive one electron through the system. A membrane or a redox couple can be used to connect the two light-absorbing systems. A potential gradient of $0 \cdot 2 \mathrm{~V}$ between each steps provides enough driving force to ensure that the back electron transfer will not take place. At $\mathrm{pH} \mathrm{7,} \mathrm{the}$ potentials will be shifted by $0.059 \mathrm{~V}$ per $\mathrm{pH}$ i.e. $0.413 \mathrm{~V}$ more negative. Such twophoton systems are a better match for solar energy spectrum since it uses photons of smaller energy.

Studies in our laboratory during the last few years have established conclusively that totally illuminated cell configuration in which both the electrodes are photoactive gives better performance (Basu et al 1985). Not only, $V_{\mathrm{oc}}^{\mathrm{ph}}$ and $I_{\mathrm{sc}}^{\mathrm{ph}}$ where $V_{\mathrm{oc}}^{\mathrm{ph}}$ is the open circuit photovoltage and $I_{\mathrm{sc}}^{\mathrm{ph}}$ is the short circuit photocurrent, are additive,

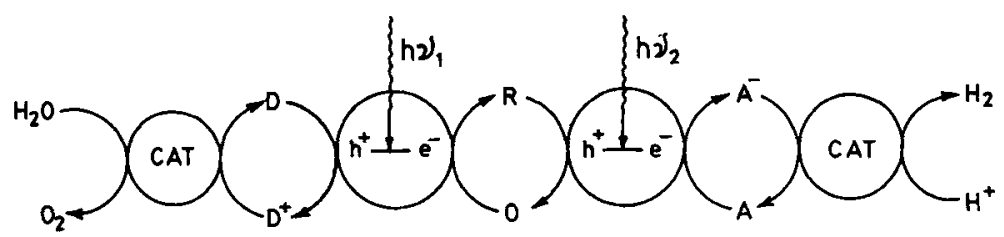

(a)

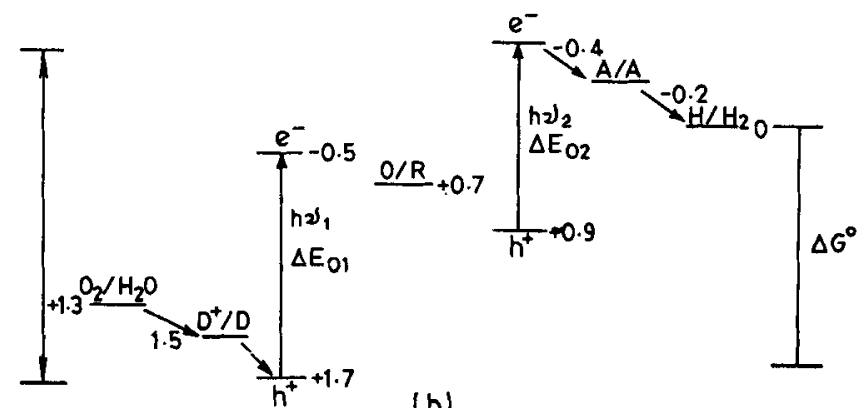

(b)

Figure 2. (a) Charge separation in two photon redox systems coupled through a membrane with donor and acceptor couples on either sides of the membrane producing $\mathrm{O}_{2}$ and $\mathrm{H}_{2}$ respectively using redox relays and catalysts. (b) Corresponding energetics scheme using semiconductors of proper band gaps and Fermi levels appropriate for photosplitting of water. 
the most important fact is that tremendous improvement in fill-factor is observed (figure 3a). Furthermore, since the process is multiphoton, solar energy engineering efficiency (SEE) also shows improvement due to better match with the solar spectrum. Figure $3 \mathrm{~b}$ presents the photoelectrochemical characteristics of a polymercoated dye-loaded ZnTPP PV-cell connected in tandem with a (PSF + EDTA) PGcell arranged in such a fashion that both the electrodes are illuminated. The two photoelectrode reactions are coupled by oxidative and reductive cycles of water splitting reaction.

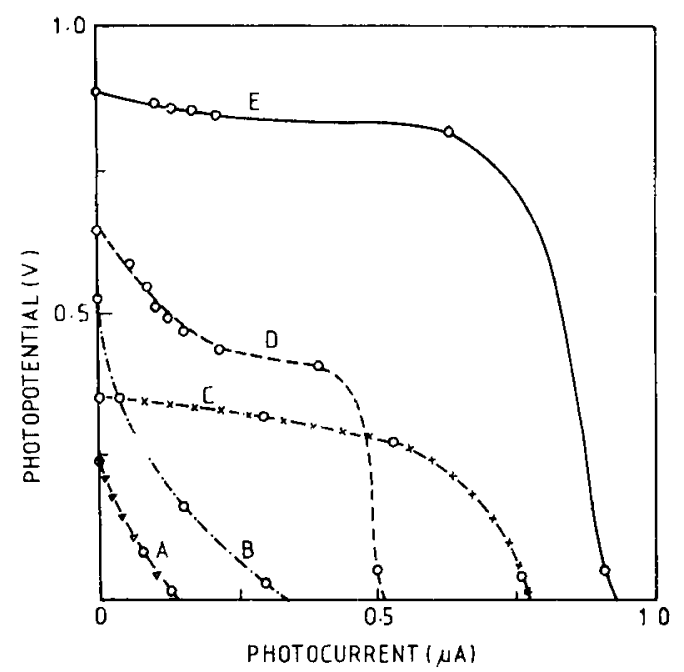

(a)

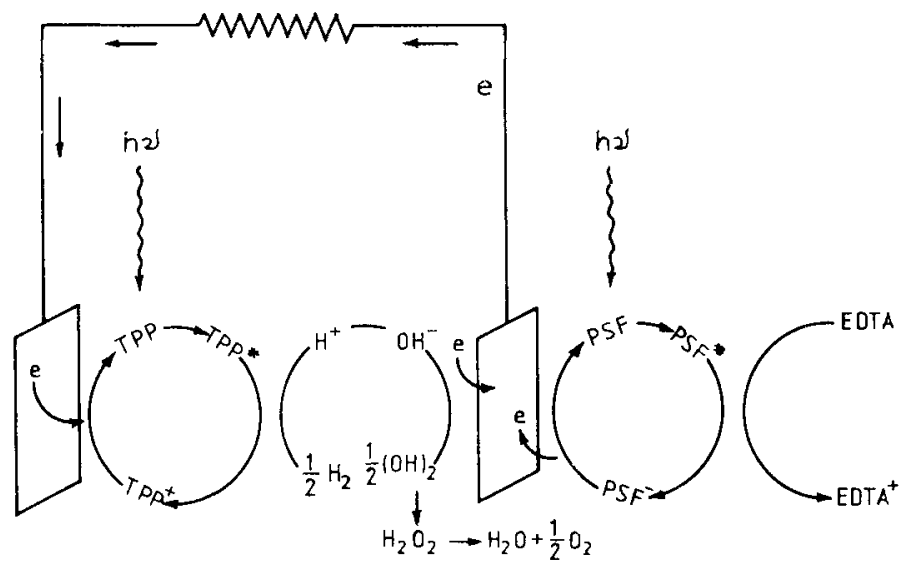

(b)

Figure 3. (a) Curreni-potental $(I \times$ V characteristics of a totally illuminated multiphoton photogalvanovoltaic (PGV) tandem cell using modified ZnTPP electrode. A: PV (photovoltatc) cell: $\mathrm{ZnTPP}$ coated $\mathrm{Pt}$ with $\mathrm{Pt}$ as counterelectrode in $\mathrm{KCl}$. ff $=0.12$. B: $\mathrm{PG}$ (photogalvanic) cell consisting of PSF + EDTA solution and $P t$ electrodes. $f f=0.19 . \quad C$ : $P V+P G$ cells in tandem. $f f=0 \cdot 5 . \quad$ D: Polymer coated $P V$ cell. $f f=0 \cdot 5 . \quad$ E: Polymer coated PVG cell. $f f=(1.66$. $(f f=$ fill factor obtained from $I \times V$ curve, $P S F=$ phenosafranine: $Z n T P P$ $=$ Zine tetraphenyl porphyrin). (b) Reaction mechanism at electrode-electrolyte interphase in the totaty illuminated tandem cell. 
One of the major problems in constructing a complete artificial photosynthetic apparatus is that not much is known about PS-II, the $\mathrm{O}_{2}$-evolving systems. During the last few years there has been a spurt of activity and considerable progress has been made in this direction. The oxygen evolution is a multistep process nec ding four flashes of light to transfer four electrons and evolve one molecule of $\mathrm{O}_{2}$. In PS-II RC (reaction centre), a special pair on photoexcitation transfers an electron to $\mathrm{Mg}$-free pheophytin and then to a quinone. Recent work has been focussed on models for PS-II RC involved in photosynthesis (Dismukes 1986).

Porphyrins and related compounds and their complexes have very similar chromophore structure and absorption characteristics as chlorophylls and hence have been widely used as photosensitizers. The redox potentials are also appropriate for participation in photo-induced electron transfer which can be studied by fluorescence quenching experiments. For such electron transfer quenching, acceptors like quinones and nitroaromatics or donors like amines, ascorbic acid, EDTA etc are used. In order to achieve uphill solar energy conversion via multielectron redox processes, it is necessary to design the best molecular structure for each step. The steps involved are (i) model system for charge separation of the primary ion pair; (ii) electron relay for prevention of back electron transfer; and (iii) multielectron redox reactions using suitable catalysts.

The systems can be examined in homogeneous solutions and the best systems can then be incorporated in bilayer membranes and liposomes to study vectorial electron transfer to mimic nature closely. Back-electron transfer may be a problem in a linked system such as porphyrin-quinones in homogeneous solutions. Organized systems are more promising. A frequent observation is that porphyrin-sensitized processes in homogeneous solutions proceed via excited triplet states while those in the reaction centres of plant photosynthesis involve electronically excited singlet states (Natarajan and Blankeship 1983).

\section{Results and discussion}

To simulate natural photosynthesis we have tried to perform experiments with (a) natural system i.e. chlorophyll extracted from spinach leaves and incorporating it in liposomes and (b) porphyrins synthesized in the laboratory.

\subsection{Experiments with natural chlorophyll}

2.1a Photophysical characterization: Chlorophyll was extracted from spinach leaves by chloroform. No attempt was made to separate $\mathrm{Chl} a$ and $\mathrm{Chl} b$. The absorption spectrum gave a broad band peaking at $430 \mathrm{~nm}$. The width and the shape of the band suggested that the solution is a mixture of other molecules also absorbing in the same region, a large components being $\mathrm{Chl} b, \lambda_{a b}=460 \mathrm{~nm}$. The excitation at $430 \mathrm{~nm}$ (absorbed by $\mathrm{Chl}$ a) generated sharp fluorescence spectrum with maximum at $\lambda_{f}=670 \mathrm{~nm}$. On excitation at $460 \mathrm{~nm}$, the fluorescence peak appeared at $\lambda_{f}=650 \mathrm{~nm}$ confirming the presence of $\mathrm{Chl} b$ in the extract. Further it was observed from the excitation spectra that phosphatidyl choline (PC-egg lecithin) could only quench the fluorescence peak at $670 \mathrm{~nm}$ when excited at $430 \mathrm{~nm}\left(\lambda_{\text {ex }}=430 \mathrm{~nm}\right)$ (figure 4). But the fluorescence peak at $650 \mathrm{~nm}\left(\hat{\lambda}_{\mathrm{ex}}=460 \mathrm{~nm}\right)$ was quenched as well as 


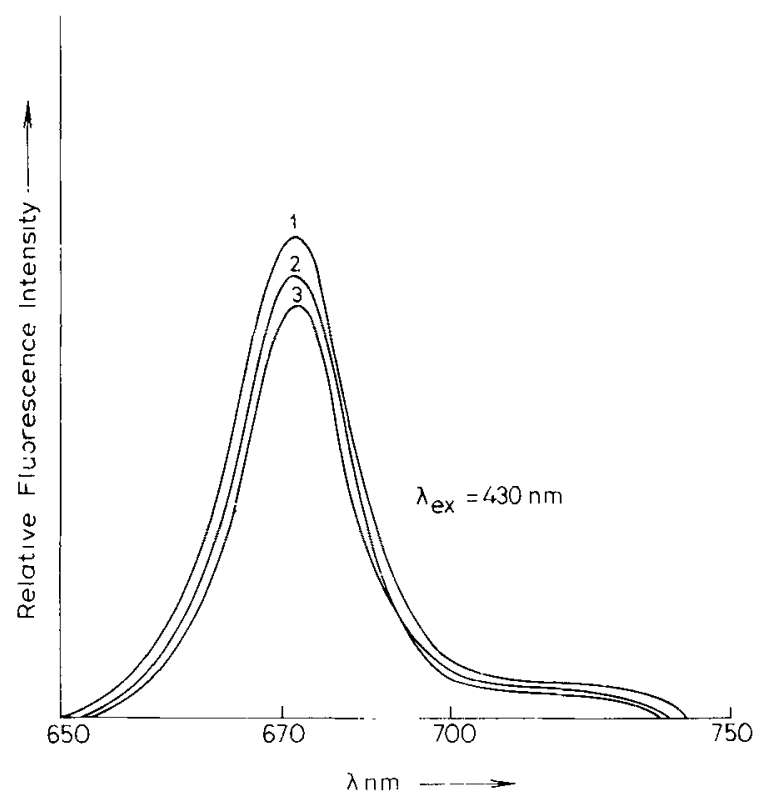

Figure 4. Fluorescence excitation spectra of purified chlorophyll extract in chloroform in the presence of egg phosphatidyl (PC). $[\mathrm{Chl}]=1 \times 10^{-6} \mathrm{M} ;[\mathrm{PC}] .1 .0 ; 2.4 \times 10^{-4} \mathrm{M}: 3$. $8 \times 10^{-4} \mathrm{M}, \lambda_{e x}=430 \mathrm{~nm}$.

red-shifted on addition of increasing concentration of PC suggesting some kind of complexation (figure 5). Since no change in the absorption spectrum of $\mathrm{Chl}$ extract was observed in the presence of PC. the shift in the fluorescence spectrum of $460 \mathrm{~nm}$ excitation band at $650 \mathrm{~nm}$, must be due to exciplex formation i.e. formation of the complex in the excited state. It appears that $\mathrm{Chl} h$ which has a $-\mathrm{CHO}$ group in place of $-\mathrm{CH}_{3}$ in $\mathrm{Chl} a$ in one of the pyrrol rings of the chloropt.yll molecule, is the site for the formation of the complex. Results also suggest that $\mathrm{Chl} a$ and $\mathrm{Chl} h$ in the extract are not coupled to each other.

2.1b Photoelectrochemistry in PC liposomes: It is already known that $\mathrm{Chl}$ is a good electron donor and the dye phenosafranin (PSF) is a good electron acceptor. An Hshaped photoelectrochemical cell (figure 6) was set up with $\mathrm{Chl}(a+b)$ incorporated in PC-liposomes in one compartment and PSF in liposome in the other compartment. Although PSF is soluble in water, it forms CT-complex with PC and hence the solution will contain some complexed and some free PSF. The two compartments were separated by a sintered glass membrane $(\mathrm{G}-4)$ which allowed transfer of $e^{-}$and $H^{+}$only. The solutions were maintained at neutral $\mathrm{pH}$ and deoxygenated by bubbling $\mathrm{N}_{2}$ gas. The cell was totally illuminated by a projector lamp tungsten $200 \mathrm{~V}, 300 \mathrm{~W}$, focussed to give $22 \mathrm{~mW} . \mathrm{cm}^{2}$ exposed area). The compartment with (PSF + PC) acted as photoanode and that with Chl-liposome as photocathode. A photovoltage $\left(V_{\mathrm{ph}}\right)$ was observed to develop with time (measured by digital multimeter) and the response decayed with time under 'off' condition. The maximum photovoltage developed was: $V_{\mathrm{ph}}^{\max }=120 \mathrm{mV}$. The $V_{\mathrm{ph}}^{r}$ also developed when each compartment was illuminated individually keeping the other in the dark. The anode 


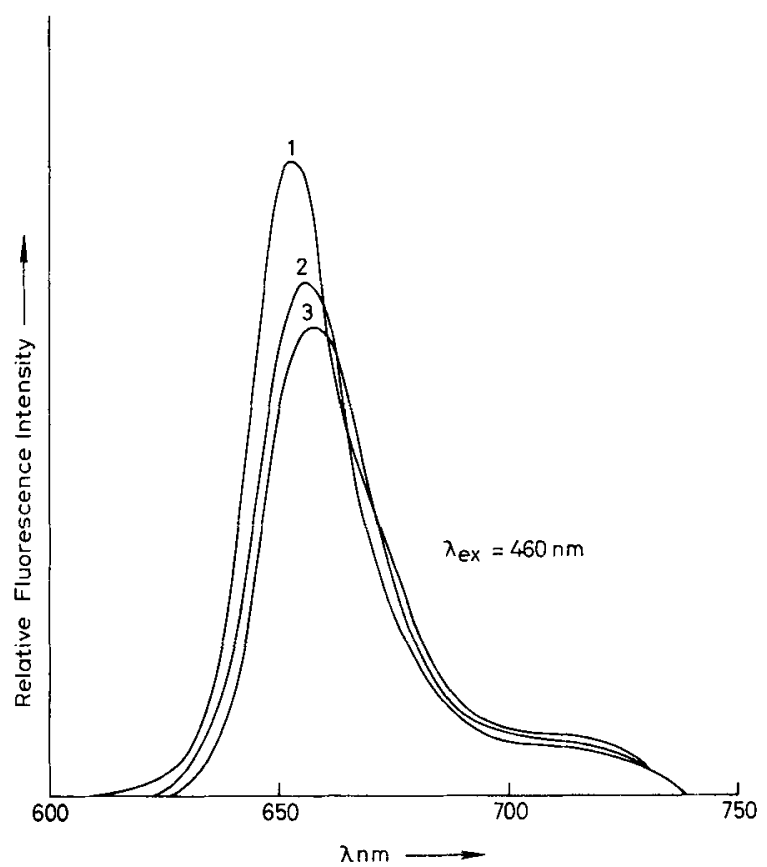

Figure 5. Same as in figure $4 ; \lambda_{\mathrm{ex}}=460 \mathrm{~nm}$.

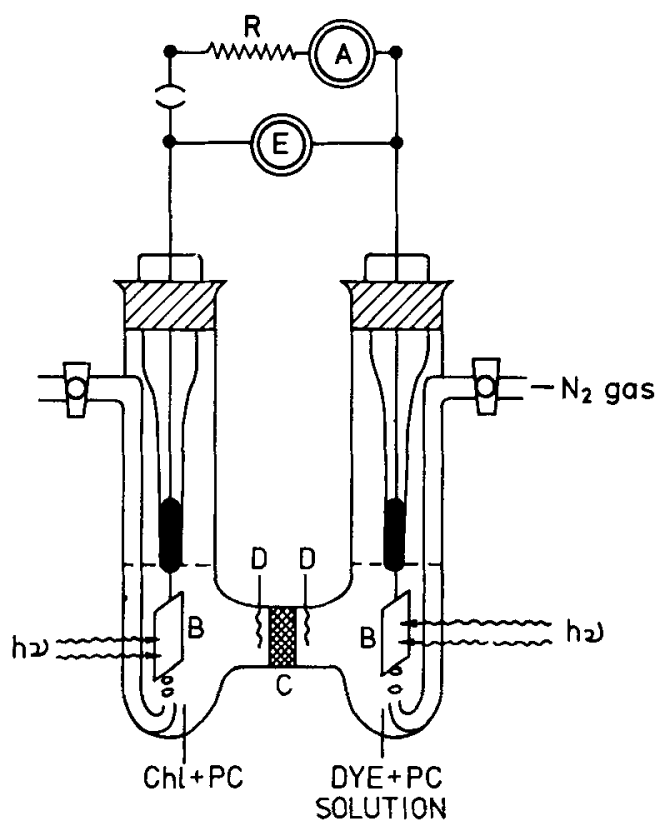

Figure 6. A schematic diagram of the experimental photoelectrochemical cell. A, multimeter; E, electrometer; R, variable resistance; B, platinum foil electrode; $C$, sintered glass membrane; D, platinum electrode. Totally illuminated system containing $\mathrm{Chl}+\mathrm{PC}$ in one side of the membrane and PSF $+\mathrm{PC}$ on the other side. Chlorophyll is $n$-type semiconductor and PSF is $p$-type semiconductor dye. 
compartment containing PSF $\left(C=1 \times 10^{-5} \mathrm{M}\right)+\mathrm{PC}$ recorded a maximum $V_{\mathrm{ph}}^{\max }=100 \mathrm{mV}$ within $40-45 \mathrm{~min}$ but when the compartment containing Chlliposomes was illuminated, only $20 \mathrm{mV}$ of photovoltage developed under similar conditions. Thus $V_{\mathrm{ph}}^{\mathrm{max}}=120 \mathrm{mV}$ for the totally illuminated cell is the sum of the two photoacts individually.

2.1c Electron transfer quenching in liposomes: Some work has also been started using liposomes in which EDTA has been introduced in the inner water pool with Chl $(a+b)$ in the liposome membrane. We are in the process of studying photosensitized electron transfer from the inner-pool to the outer-pool. With chlorophyll incorporated within the vesicle and EDTA in the inner water-pool of the vesicle, fluorescence quenching by 1,5-anthraquinone disulphonic acid present in the outer water-pool was observed. No quenching was observed in the absence of EDTA in the inner-pool. This observation establishes that EDTA is the electron donor and initiates vectorial electron transfer by blocking the hole left behind on excitation of Chl molecules located within the lipid bilayer as in natural photosynthesis. Considering the size of the vesicles which normally have the dimensions, outer diameter of approximately $200 \AA$ and vesicle thickness of approximately $50 \AA$, it appears that more than one $\mathrm{Chl}$ molecules participate in transporting the electron from the inner boundary to the outer boundary. Direct electron transfer at such large distances is not possible. For vesicle formation, a concentration of $10^{-4} \mathrm{M}$ PC was used with $[\mathrm{Chl}]=10^{-5} \mathrm{M}$, which corresponds to roughly 300 molecules of $\mathrm{Chl}$ per liposome. Therefore a scheme for electron transfer from inner water-pool to outer-pool can be represented as given in figure 7 .

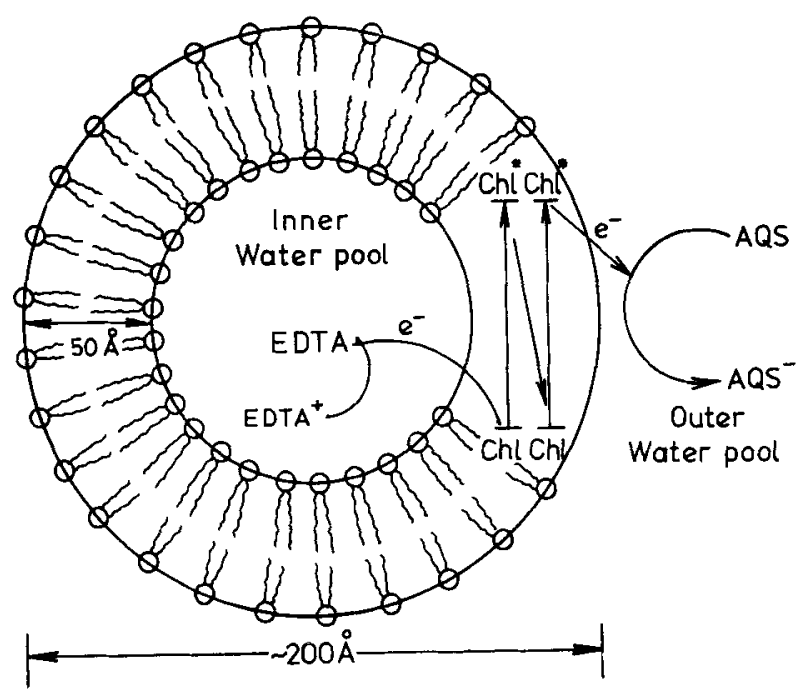

Figure 7. Chlorophyll (photosensitizer) incorporated in phosphatidyl choline (PC) vesicle with EDTA (electron donor) in the inner pool and 1,5-anthraquinone disulphonate AQS (electron acceptor) in the outer pool, promoting vectorial electron transfer from EDTA to AQS. 


\section{Experiments with synthetic porphyrins in micelles}

Porphyrins are analogues of chlorophylls and can give more stable systems compared to chlorophylls. Work on the synthesis of various substituted and metallated porphyrins is in progress in our laboratory and this experience has helped us to synthesize water soluble tetrakis (4-phenyl-sulphonate) porphyrin, $\mathrm{TPP}\left(\mathrm{SO}_{3}^{-}\right)_{4}$. The compound was purified by passing through a sephadex column. The absorption and emission spectra were recorded.

Since this porphyrin molecule contains 4 bulky $-\mathrm{SO}_{3} \mathrm{H}$ groups which are dissociated to give 4-1onised $\mathrm{SO}_{3}^{-}$groups, the molecule contains 4 units of negative charge. If incorporated into a micelle, the molecule lies on the surface only and imparts highly negative surface potential to the micelle which can be gainfully used in the step for charge separation in a suitably organized system preventing back electron transfer.

The excited state lifetime $\tau$ of TPP $\left(\mathrm{SO}_{3}^{-}\right)_{4}$, in the neutral micelle of triton $\mathrm{X}-100$ was studied by single photon counting technique. The data are given in table 1 . After initial decrease, the value of $\tau$ starts increasing again at $[$ triton $X-100]=2.5 \times 10^{-4} \mathrm{M}$. This value corresponds to $\mathrm{CMC}$ (critical micelle concentration) of triton $\mathrm{X}-100$. In the premicellar region, decrease in lifetime may be of general nature caused by

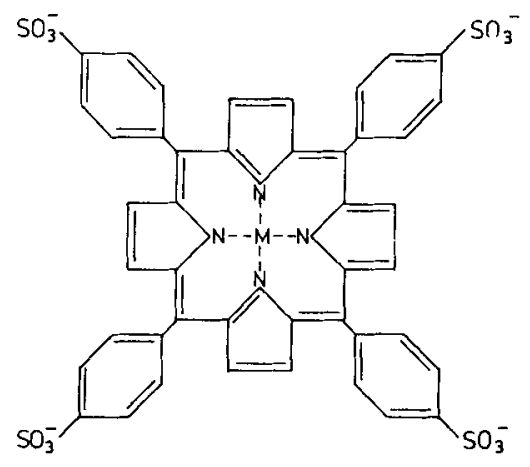

Figure 8. Structure of (tetrakis (4-phenyl-sulphanato) porphyrin): $\mathrm{TPP}\left(\mathrm{SO}_{3}^{-}\right)_{4}$.

Table 1. Variation in the fluorescence lifetime $\tau$ nanosecond, of $\mathrm{TPP}\left(\mathrm{SO}_{3}^{-}\right)_{4}$ in micellar medium, in absence and on addition of various concentrations of the surfactant Triton X-100. The CMC represents the critical micelle concentration of triton X-100.

\begin{tabular}{ll}
\hline$[$ Triton X-100] M & $\begin{array}{c}\tau \\
\text { in ns }\end{array}$ \\
\hline 0 & 10.25 \\
$1 \times 10^{-5}$ & $10 \cdot 19$ \\
$4 \times 10^{-5}$ & $10 \cdot 16$ \\
$2.5 \times 10^{-4}$ & $10.85-\mathrm{CMC}$ \\
$1.0 \times 10^{-3}$ & 12.27 \\
$7.5 \times 10^{-3}$ & 12.08 \\
$1.0 \times 10^{-2}$ & 12.62 \\
\hline
\end{tabular}


interaction between surfactant molecule and $\mathrm{TPP}\left(\mathrm{SO}_{3}^{-}\right)_{4}$ molecules. Above $\mathrm{CMC}$, the molecule being highly charged and bulky, must reside at the micelle-water interface. This brings about a certain amount of rigidity in the molecule and consequently the rate of internal conversion $k_{\mathrm{IC}}$ of photoexcited species must decrease. The inhibition of 'loose bolt' effect leads to the enhancement of mean life of the molecule. The system is under further study.

\section{Acknowledgements}

We acknowledge with thanks the financial assistance from the Department of NonConventional Energy Source, Government of India. We thank Dr Jyotsna Basu for help in the synthesis of the porphyrin compound and Dr Papiya Nandi for useful discussion.

\section{References}

Calvin M 1979 Energy Re's. 373

Fendler J H 1983 I. Chem. Edn. 60872

Fendler J H 1985 J. Phys. Chem. 892730

Basu J. Chatterjec A B and Rohatgi-Mukherjee K K 1985 Indian J. Chem. A24 550

Dismukes G C 1986 Photochem. Photobiol. 4399

Natarajan L V and Blankeship R F 1983 Photechem. Photohiol. 37329 